\title{
Desperdicios de alimentos (DA) en sodas concesionadas de una Universidad Pública en Heredia, Costa Rica
}

\section{Food waste (FW) in concessioned sodas of a Public University in Heredia, Costa Rica}

Julián Rojas-Vargas ${ }^{1}$, Yanory Monge-Fernández², Kateryn Fernández- Hidalgo ${ }^{3}$

Rojas-Vargas, J; Monge-Fernández, Y; Fernández-Hidalgo, $\mathrm{K}$. Desperdicios de alimentos (DA) en sodas concesionadas de una Universidad Pública en Heredia, Costa Rica. Tecnología en Marcha. Vol. 33-1. Enero-Marzo. Pág 152-164.

doi https://doi.org/10.18845/tm.v33i1.5029

1 Lic. Biología Tropical. UNA Campus Sostenible. Universidad Nacional. Heredia, Costa Rica. Correo electrónico: julian.rojas.vargas@una.cr. (iD https://orcid.org/0000-0003-2592-2872

2 Bach. Biología Tropical. UNA Campus Sostenible. Universidad Nacional. Heredia, Costa Rica. Correo electrónico: yanorymf@hotmail.com.

3 Bach. Género y Desarrollo. UNA Campus Sostenible. Universidad Nacional. Heredia, Costa Rica. Correo electrónico: kfernandez.h@gotmail.com. 


\title{
Palabras clave
}

Pérdida; desperdicio; alimentos; residuos orgánicos.

\section{Resumen}

Alrededor de 815 millones de personas padecen de hambre en el mundo y de estos 39 millones de personas sufren de este problema en América Latina y el Caribe. Cerca de un tercio de los alimentos producidos en el mundo se pierden o son desperdiciados. Según la FAO, estas cifras van en aumento, dejando en evidencia que el cambio climático, las malas prácticas en la agricultura y el desperdicio de alimentos pone en peligro la seguridad y la soberanía alimentaria. Por eso, el objetivo de este trabajo fue determinar la pérdida y desperdicio de alimentos (PDA) con respecto a la producción diaria de alimentos, para la identificación de estrategias que minimicen o solucionen el PDA en las sodas del Campus Omar Dengo. Se midió el desperdicio de alimentos en las distintas sodas del Campus Omar Dengo de la Universidad, con una metodología estandarizada establecida por la Red Costarricense para la Disminución de Pérdida y Desperdicio de Alimentos y la iniciativa de SAVE FOOD®. Se obtuvo diferencias significativas en los valores de PDA generados por las sodas. Características como el tipo de población y las buenas prácticas en la cocina causan menos desperdicios de alimentos. Se concluye que se debe trabajar con los usuarios de las sodas mediante campañas de concientización sobre el tema de desperdicios para disminuir los porcentajes tan altos presentados en el área de devolución en platos.

\section{Keywords}

Lost; waste; food; organic waste.

\begin{abstract}
Around 815 million people suffer from hunger in the world and of these 39 million people suffer from this problem in Latin America and the Caribbean. About one third of the food produced in the world is lost or wasted. According to the FAO, these figures are on the rise, showing that climate change, bad practices in agriculture and food waste jeopardize food security and sovereignty. Therefore, the objective of this work was to determine the loss and waste of food (PDA) with respect to the daily production of food, for the identification of strategies that minimize or solve the PDA in the sodas of the Omar Dengo Campus. Food waste was measured in the different sodas of the Omar Dengo Campus of the University, with a standardized methodology established by the Costa Rican Network for the Reduction of Food Loss and Waste and the SAVE FOOD® initiative. Significant differences were obtained in the PDA values generated by the sodas. Characteristics such as the type of population and good practices in the kitchen cause less food waste. It is concluded that you must work with the users of the sodas through awareness campaigns on the subject of waste to reduce the percentages so high presented in the area of return in dishes.
\end{abstract}

\section{Introducción}

Los últimos datos indican que el número de personas que padecen hambre en el mundo continúa en aumento, esto evidencia la necesidad de que se deben tomar medidas en el tema e implementar acciones efectivas, es preocupante el aumento de los niveles de desnutrición 
infantil, uno de cada cuatro niños en el mundo padece de retraso en el crecimiento. (150,8 millones, de niños menores de 5 años afectados por retraso del crecimiento.)

Costa Rica también presenta esta problemática de subalimentación, 5,6\% de la población la sufre. Además, el caso opuesto es la obesidad, que también presenta un alza. Por esa razón a través de la FAO se han implementado diversos programas y proyectos nacionales e internacionales con la idea de poder cumplir con el objetivo de desarrollo Sostenible N²: cero hambre, con el fin de garantizar la seguridad alimentaria, mejora de la nutrición y promover la agricultura sostenible. En el caso de Costa Rica, se conformó la Red Costarricense para la Disminución de Pérdidas y Desperdicios de Alimentos en conjunto a la iniciativa mundial SAVE FOOD $®$.

La idea de este artículo es evidenciar los diversos resultados obtenidos a través de la medición y diagnóstico de las pérdidas de alimentos que se da en cuatro sodas de la Universidad Nacional, destacando también las buenas prácticas de algunas sodas en cuanto a la manipulación de los alimentos.

Se realizó una medición de los diversos alimentos, cocinados y sin cocinar en los distintos tiempos de preparación de alimentos, desayuno y almuerzo que representan el movimiento más grande de la población estudiantil y su acceso a servicios en las sodas, así como la medición de alimentos desperdiciados en plato por parte de la población estudiantil.

Se presentan resultados obtenidos en cuanto a la pérdida de desperdicios de alimentos para las sodas en desperdicios de platos, precios y sobre todo cuántas personas se pudiesen alimentar con esa comida. A través del análisis de los diversos datos obtenidos, se presentan para una mejor comprensión cuadros y gráficos explicitando la información que se obtuvo.

La intención de este análisis y trabajo es poder brindar recomendaciones, conclusiones ante el desperdicio de alimentos, estrategias y opciones que sean de beneficio tanto para los concesionarios de las sodas y para la Universidad Nacional (funcionarios y estudiantes) logrando todos en conjunto un nivel de sensibilización y reducción de los desperdicios de alimentos.

\section{Marco Teórico}

Pérdida y Desperdicio de alimentos: Parte del compromiso a nivel internacional de la Organización de las Naciones Unidas para la Alimentación y la Agricultura, (FAO), a través de la amplia experiencia que reúne, sus conocimientos y compromisos para afrontar los desafíos que relacionan la alimentación y la agricultura de la población mundial. Se sustentan sus bases en políticas estratégicamente elaboradas para aumentar la capacidad de apoyar a los gobiernos, asociados y partes interesadas en temas y asuntos de alimentación y agricultura.

En el marco de los Objetivos del Desarrollo Sostenible (ODS), la FAO, estableció compromisos para afrontar la problemática de la pérdida y desperdicio de alimentos, vinculado esto a la problemática del hambre, agotamiento de los recursos renovables, calentamiento global.

El proyecto de disminución de pérdidas y desperdicios de alimentos en América Latina y el Caribe, busca reducir a la mitad las pérdidas y desperdicios de alimentos per cápita en 2025, en donde se debe entender que las pérdidas de alimentos son y suceden durante la producción, postcosecha, almacenamiento y transporte de los alimentos, por otra parte, las pérdidas alimentarias son los productos agrícolas, ganaderos o pesqueros destinados al consumo humano pero que al final no son consumidos. Mientras que, por desperdicio de alimentos, se puede entender que son los alimentos que fueron desechados o utilizados de manera alternativa, esto 
se produce en el proceso de distribución y el comercio, durante la preparación y consumo final, además de quienes deciden desechar los alimentos que aún tienen valor. [1]

Además, el trabajo realizado en las distintas sodas de la Universidad Nacional, pretenden dar los diversos resultados obtenidos sobre el desperdicio de alimentos en las diversas sodas, además de que este representa la disminución de masa y calidad de un producto intencionado para alimentación humana.

Objetivos Desarrollo Sostenible: Con la idea de crear transformaciones positivas en diversos aspectos a nivel mundial, se creó en el 2015 una nueva agenda de desarrollo sostenible, que se pone en marcha en conjunto de objetivos, con la idea de erradicar el hambre, asegurar la alimentación, mitigar el impacto del calentamiento global, entre otros.

La implementación a nivel mundial, de estos objetivos y sus metas, se esperan un cumplimiento para el 2030. Un ejemplo, claro e importante es el Objetivo 2, "Poner fin al hambre, lograr la seguridad alimentaria, mejorar la nutrición y promover la agricultura sostenible". En donde su meta principal, entre los grandes desafíos es que se logre una seguridad alimentaria, que aborde todos los factores de productividad, resiliencia de los sistemas alimentarios, uso sostenible de la biodiversidad y los recursos. Ya que pretende a través de las metas de objetivos como este y en colaboración con la FAO promover diversos proyectos que tienen como fin una seguridad alimentaria.

Por ello, se crea y adopta un proyecto y estudio de la FAO en la que participan diferentes gobiernos y asociados, llamado pérdidas y desperdicio de alimentos (PDA). Costa Rica también se ha involucrado. A nivel de América Latina y el Caribe, se avanza coordinadamente, esa es una de las muchas estrategias que pretenden alcanzar el cumplimiento de los Objetivos de Desarrollo Sostenible. [3]

Seguridad e inseguridad alimentaria: Parte de la crisis que se vive a nivel mundial con la mala alimentación, son la inseguridad alimentaria y la desnutrición, que perjudican los medios de vida y los sistemas alimenticios. La falta de medidas óptimas para erradicar el hambre, medios y vías que promuevan una seguridad en los sistemas de producción alimentaria sostenible y prácticas agrícolas resilientes. En [5], se destaca como la inseguridad alimentaria va en aumento, que existen situaciones específicas en donde esta problemática presenta un riesgo a la seguridad de brindarle el acceso a la alimentación a todas las personas a nivel mundial. Es debido a lo anterior que es indispensable crear estrategias, objetivos y metas a través de los diversos proyectos y programas de la FAO.

Residuo: Según [6] un residuo corresponde a material sólido, semisólido, líquido o gas, cuyo generador o poseedor debe o requiere deshacerse de él, y que puede o debe ser valorizado o tratado responsablemente o, en su defecto, ser manejado por sistemas de disposición final adecuados. (p.14)

\section{Objetivo general}

Determinar la pérdida y desperdicio de alimentos (PDA) con respecto a la producción diaria de alimentos, para la identificación de estrategias que minimicen o solucionen el PDA en las sodas del Campus Omar Dengo de la Universidad Nacional. 


\section{Metodología}

Sitio de estudio

El presente estudio se realizó en las cocinas de cuatro sodas en el Campus Omar Dengo de la Universidad Nacional, en los siguientes periodos de muestreo: Soda N¹ marzo 2018. Soda 2 abril 2018. Soda 3 mayo 2018. Soda 4 agosto 2018 (figura 1).

En la mayoría de las sodas se trabajó desde las seis de la mañana a las dos de la tarde, por lo que se incluyó únicamente los alimentos preparados para el desayuno y para el almuerzo. Se realizó durante tres días en cada soda tratando de que fuera en la misma semana según se recomienda y se tomaron en cuenta el proceso desperdicio en tres áreas específicas y la producción diaria de alimentos.

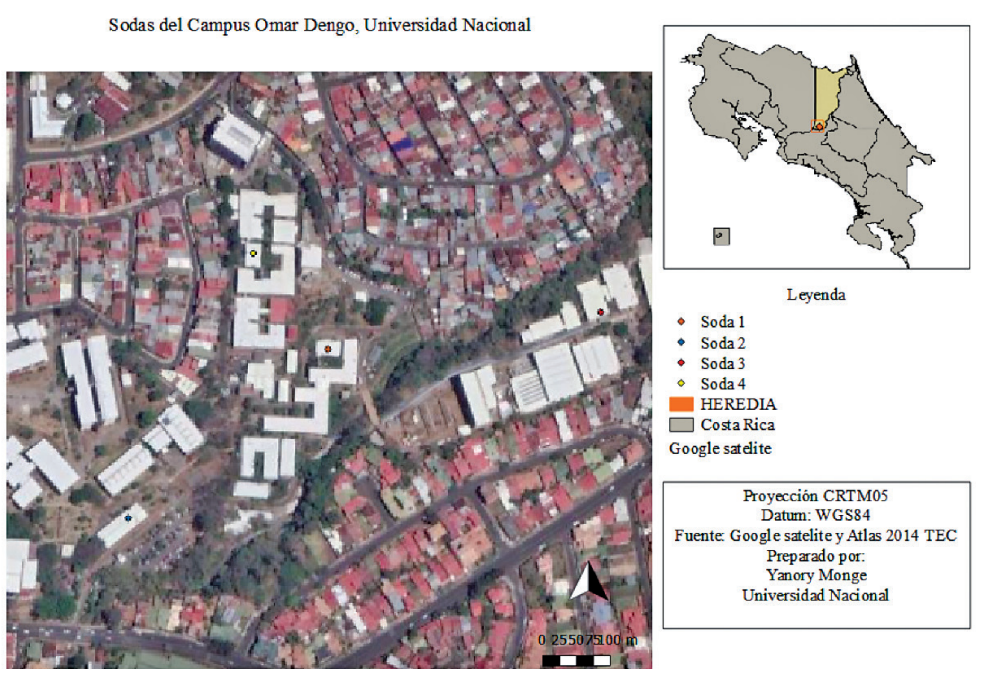

Figura 1. Ubicación de las sodas evaluadas en el Campus Omar Dengo

\section{Recolecta y procesamiento de desperdicios}

Se utilizó la Guía de mediciones de cocinas institucionales y comerciales establecida por la Red Costarricense para la Disminución de Pérdida y Desperdicio de Alimentos y la Save Food Costa Rica (Red Costarricense para la disminución y PDA, 2017). En cada soda se colocó cuatro recipientes plásticos para cada una de las áreas utilizadas en el cálculo de Pérdida y Desperdicio de Alimentos (PDA); área de bodega, área de preparación y área de residuos de platos (figura 2).
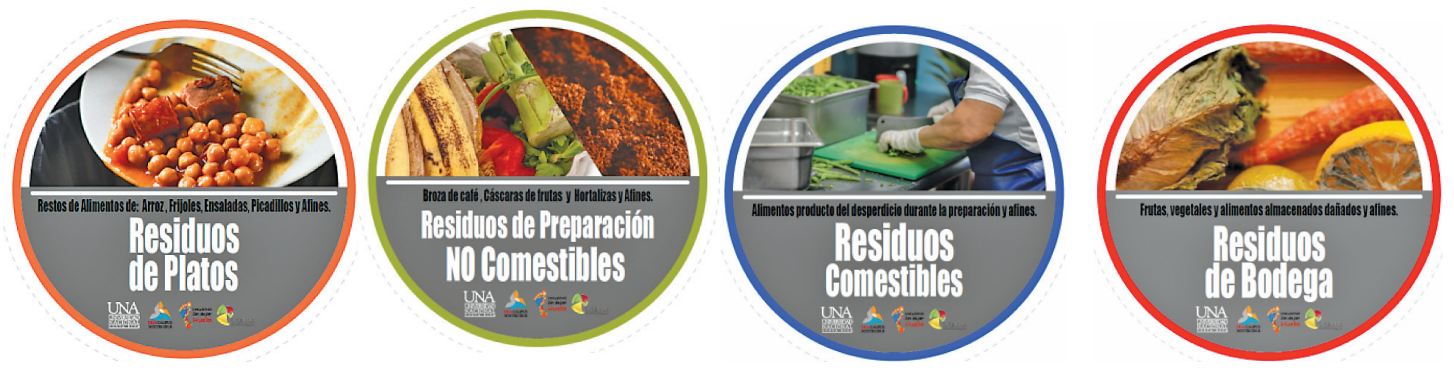

Figura 2. Pegatinas para la separación y clasificación de los desperdicios por áreas. Fuente: UNA Campus Sostenible. 
En el área de bodega se contempló todos los productos sacados de alacenas, cámaras de frío, cuartos de refrigeración, cestas de vegetales, bodegas y almacenes. Los cuales no pueden utilizarse para cocinar debido a que están dañados, vencidos o con algún otro desperfecto, se incluyó frutas, hortalizas, verduras, lácteos, embutidos entre otros.

En el área de preparación se contempló todos los alimentos que, a la hora de cocinar, picar, cortar, hornear, freír o mezclar se quemó, dañó, contaminó, se ensució o no cumplía características óptimas para ser servidas y consumidas por usuarios. Además, se incluyeron los desperdicios que se dan a la hora de trocear algunos alimentos como carnes y alimentos que por su estética no pueden ser servidos (figura 3). También en esta área se incluyó los residuos orgánicos no comestibles que son los que corresponden a cáscaras de vegetales, cáscaras de huevo, algunas semillas, huesos, y residuos de alimentos afines que no se pueden consumir.

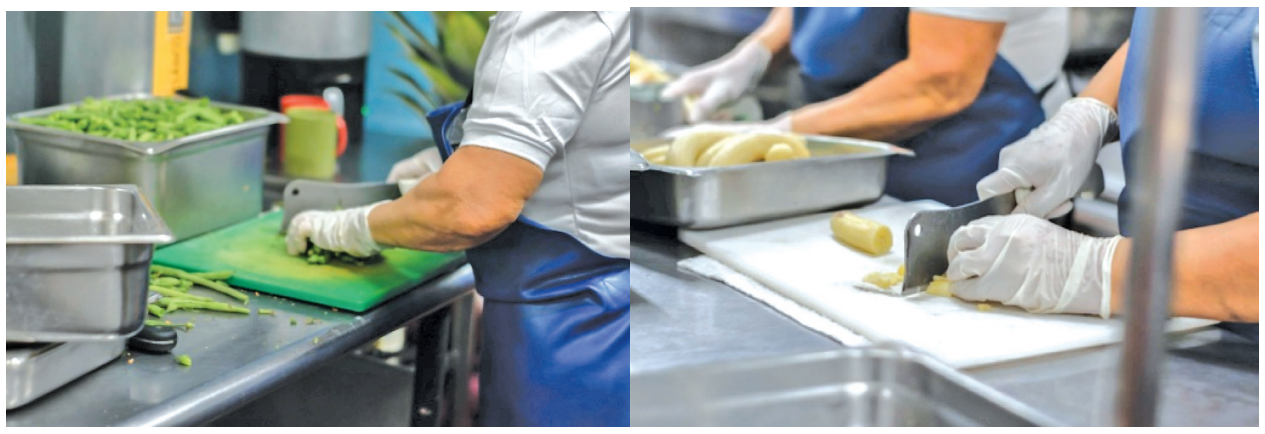

Figura 3. Proceso de picado de los alimentos

Finalmente, en el área de residuos de platos se incluyó todos los desperdicios dejados por los usuarios de las sodas, para lo cual se colocó recipientes donde los usuarios depositaban el desperdicio o bien en la cocina donde una de las ayudantes se encargaba de depositar este residuo en un recipiente antes de lavar los utensilios (figura 4).
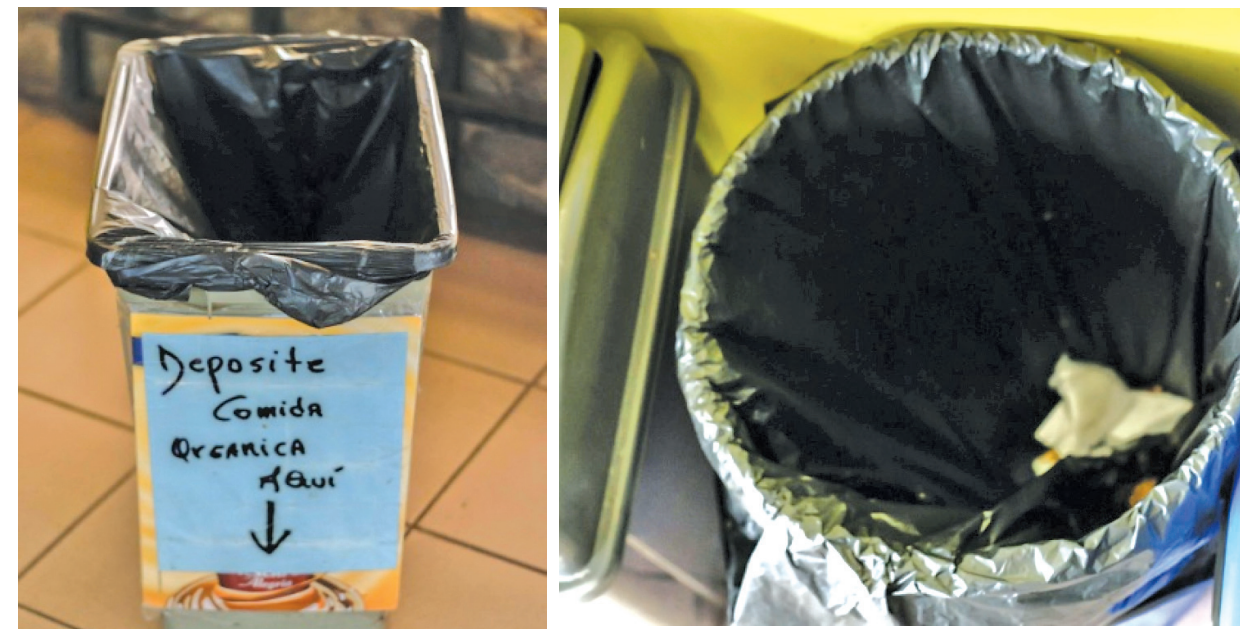

Figura 4. Recipiente colocado para los residuos de platos de los usuarios

Todos estos recipientes se colocaron desde las seis de la mañana hasta aproximadamente las dos de la tarde donde fueron recolectados y pesados en una balanza electrónica. Algunos fueron pesados hasta el final del proceso, sin embargo, recipientes de residuos no comestibles los cuales se generan en grandes cantidades se pesaron a lo largo del proceso (figura 5). 

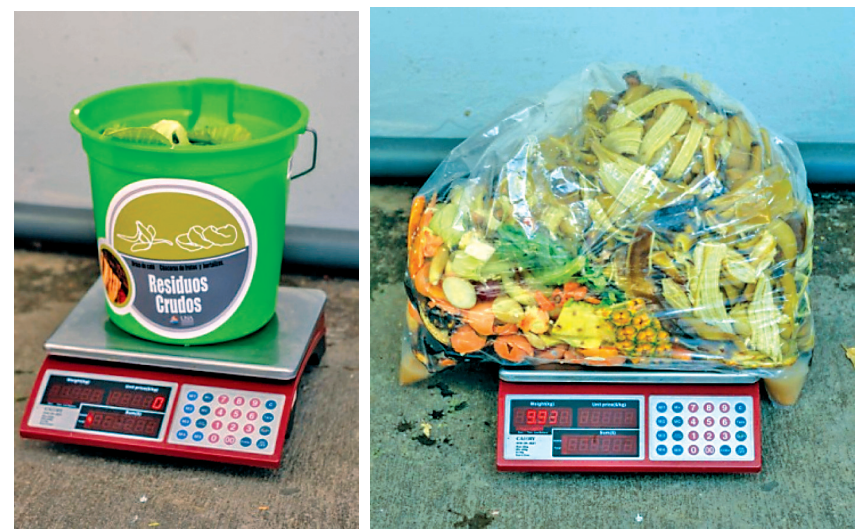

Figura 5. Pesado de baldes y bolsas del área de bodega y preparación no comestible

\section{Producción diaria de alimentos}

Se contabilizó la producción diaria de alimentos a lo largo de los tres días de muestreo por soda. Para esto se tomó en cuenta el peso de los productos o alimentos iniciales que salen de bodegas y afines en estado crudo, semipreparado o preparado pero que se terminan de preparar o cocinar para servir a los usuarios de las sodas. Estos datos se anotan en una plantilla y a los productos crudos se aplicó un factor de conversión para tener el valor del producto cocinado (Red Costarricense para la disminución y PDA, 2017). Esto se realizó con todos los alimentos tanto para el desayuno como para el almuerzo (figura 6). Posteriormente pasada la hora de demanda del desayuno o del almuerzo se anota el peso del producto a alimento final que no fue servido ni consumido pero que puede almacenarse para consumirlo posteriormente siguiendo ciertas normas de inocuidad.
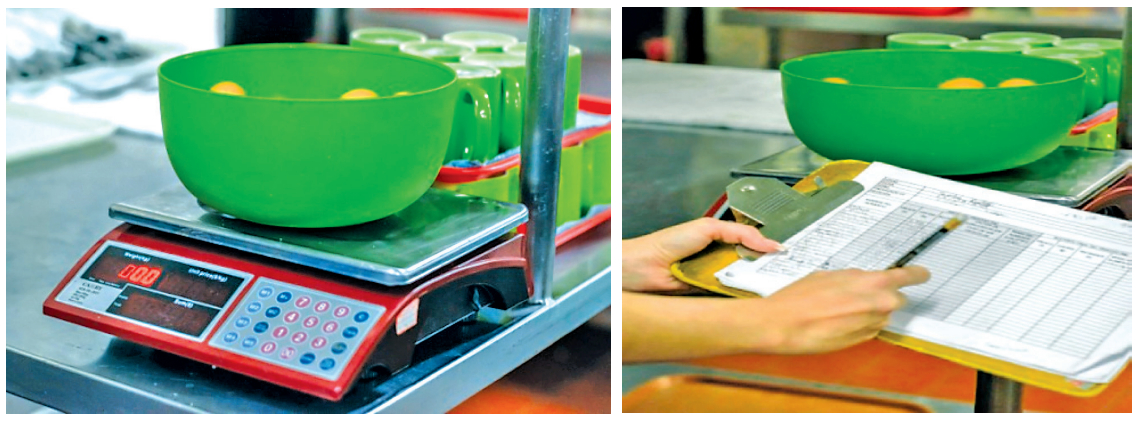

Figura 6. Pesado de los alimentos y anotación en plantillas.

Análisis de la información

Con base en los datos recolectados se calculó la producción diaria de alimentos para cada producto y una sumatoria total que da la producción diaria total $\left(\mathrm{PD}_{T}\right)$ (ecuación 1), el porcentaje de PDA con respecto a la producción diaria (\%PDA ${ }_{P D}$ ) en cada día (ecuación 2) y el porcentaje de PDA en bodega $\left(\% P D A_{B}\right)$, preparación $\left(\% P D A_{P}\right)$ y residuos de platos $\left(\% P D A_{R P}\right)$ (ecuaciones 3,4 y 5 respectivamente).

$$
P D_{T}=\text { Sumatoria }\left(P_{i}-P_{f}\right) \quad(1) \quad \% P D A_{P D}=\frac{F}{P D} * 100
$$

Donde $P_{i}=$ Peso del producto inicial $(\mathrm{kg}), \mathrm{P}_{\mathrm{f}}=$ Peso del producto final (kg). F= El PDA/día (kg), $\mathrm{PD}=$ Producción diaria/ día $(\mathrm{kg})$ 


$$
\% P D A_{B}=\frac{C_{B}}{E} * 100 \quad \text { (3) } \% P D A_{P}=\frac{C_{P}}{E} * 100 \quad \text { (4) } \% P D A_{R P}=\frac{C_{R P}}{E} * 100
$$

Donde $C_{B}=$ Masa de residuos en el área de bodega, $C_{P}=$ Masa de residuos en el área de preparación, $\mathrm{C}_{\mathrm{RP}}=$ Masa de residuos en el área de residuos de platos. E=Sumatoria total de pérdidas de alimentos por día (masa de residuos en las tres áreas kg).

Cada aspecto se calculó por día y se promedió por tres para tener el resultado general de cada soda, y se mostraron los datos mediante gráficos comparativos. Se realizo un análisis de varianza (ANOVA) para determinar si existen diferencias significativas entre los datos mediante el programa estadístico R Studio 4.6.

\section{Resultados y discusión}

Los resultados encontrados indican que existen diferencias significativas en el PDA obtenido en las cuatro sodas evaluadas $(F=7.526, g . l=3, p$-value=0.0102), las cuales por características propias como personal, equipamiento de la cocina, usuarios que la visitan entre otros aspectos, son muy diferentes entre sí, además el manejo de los alimentos según cada concesionario. De acuerdo con [2], [16] y [17] las buenas prácticas en el almacenaje, uso y preparado de los alimentos pueden contribuir a un menor desperdicio de estos, situación que pudo ser observada en la soda 2 quienes tuvieron un porcentaje de PDA más bajo $(8.19 \mathrm{~kg})$ en comparación con la soda 4 (18.57 kg) (figura 7).

Además, el promedio general de PDA obtenido en este estudio fue de $11.97 \mathrm{~kg} \pm 4.56$. Con base en este resultado se pudo calcular que se desperdicia $47.88 \mathrm{~kg}$ de comida en todas las cuatro sodas del Campus Omar Dengo, lo que corresponde a una estimación de $10533.6 \mathrm{~kg}$ de desperdicios al año, de acuerdo con los 220 días de servicio de las sodas en la universidad aproximadamente. Además, un plato servido en la soda pesa alrededor de $0.680 \mathrm{~kg}$ (que corresponde al gramaje del peso del plato establecido en la Universidad Nacional) se tiene que al año se pierden 15490 platos de comida (Almuerzos) que pueden representar en pérdidas económicas de hasta ф23 235000.

Estos datos indican que el problema de desperdicios de alimentos en la universidad es considerable y se ve reflejado en el gasto económico al que incurren las sodas para preparar estos alimentos. Cada plato tiene un costo económico, corresponde darle un manejo eficiente a los alimentos y a la vez concientizar a la población universitaria. Aunque no se tiene un dato de cuanto es la pérdida en términos económicos del desperdicio de alimentos, para [11] y con base en datos de la FAO se estima que en el 2016 los países con alto desarrollo industrial presentaban pérdidas de hasta $\$ 680000$ millones y en países en vías de desarrollo alrededor de \$310 000 millones. Cifras muy cuantiosas que indican una mala gestión y control en el uso de los alimentos. Sumado a esto se encuentra el coste ambiental y social de los desperdicios de alimentos.

Cabe recalcar que cuando se realizó el muestreo en la soda 4, la soda 3 se encontraba cerrada por problemas administrativos. Por lo que parte de los usuarios de esta soda se trasladaron a otras sodas y esto pudo causar el aumento en los residuos orgánicos en la soda 4, situación que puede ser corroborado por los registros diarios de residuos orgánicos crudos y cocinados que maneja UNA Campus Sostenible el cual aumentó significativamente en esta soda en el mes de agosto [7]. Sin embargo, esta soda por si misma genera gran cantidad de residuos crudos que corresponden a cáscaras de frutas y verduras y demás partes no comestibles, lo que indica que no se está aprovechando de la mejor manera los alimentos, o hay malas prácticas en la cocina. 


\section{PDA en cada soda evaluada}

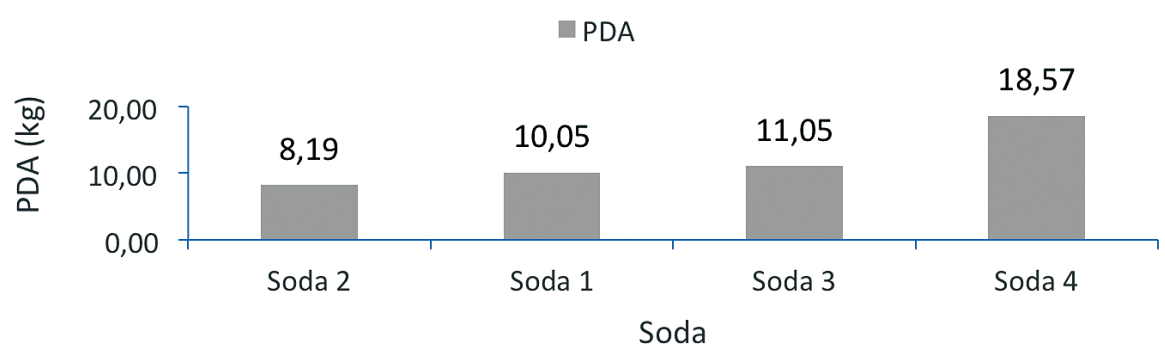

Figura 7. Pérdida y desperdicio de alimentos (PDA) promedio por soda

A nivel de cada área de las cocinas, se encontró que no hay diferencias significativas en el PDA obtenido en el área de bodegas de las cuatro sodas evaluadas $(F=4.021, \mathrm{~g}$. I=3, p-value=0.0513) así como en el área de preparación $(F=3.648, g . l=3, p$-value=0.0636) (figura 8). La soda 1 obtuvo un porcentaje de PDA muy bajo en el área de bodega (4.90\%), en comparación con las otras sodas y en comparación con el valor teórico estipulado (21\%). Se debe aclarar que este valor teórico fue calculado por [8] para hoteles y restaurantes, lugares donde se maneja mayor cantidad de alimentos para más personas, a diferencia de las sodas de la Universidad Nacional, sin embargo, se hace la comparación por ser el único valor del que se tiene disponible para Costa Rica.

Una de las características que se pudo observar como parte del manejo de los alimentos en la cocina de la soda 1 fue la tendencia a comprar alimentos frescos para ser utilizados el mismo día, principalmente por el poco espacio en las alacenas o bodegas, por lo tanto, los productos rara vez salían deteriorados y se lleva un buen control de vencimientos. De acuerdo con [9] y [10] muchos productos son consumibles, pero por errores en los sistemas de almacenamiento o en el control de fechas de caducidad se pueden perder cantidades importantes. La soda 2 presentó mayor desperdicio de alimentos en esta área de la cocina, es probable que esto se deba a un control más bajo en los vencimientos y estados de los alimentos en bodega principalmente por la gran cantidad de alimentos que compra la soda para una población bastante grande de usuarios, por lo que se dificulta el control de los productos. Además, el promedio general obtenido en esta área $(17.61 \pm 11.23)$ se encuentra algo cercano al valor teórico estipulado (cuadro 1).

De igual forma en el área de preparación ninguna de las sodas presentó un porcentaje de desperdicio tan alto como el valor teórico estipulado (45\%), sin embargo, la soda 1 presentó el porcentaje más alto (29.34\%) y la soda 3 no obtuvo ningún desperdicio de alimentos en esta área (figura 8). La razón por la que ésta última soda no obtuvo PDA puede deberse a buenas prácticas de inocuidad y de aprovechamiento del alimento. De acuerdo con [11] la falta de formación de las personas que manipulan la comida y el no planear bien cómo se van a preparar los alimentos conduce a que se desperdicie ciertos trozos de los alimentos que podrían ser aprovechados. Además, esta soda presenta un control estricto de las porciones que cocinan tratando de aprovechar al máximo posible el alimento anotando en plantillas los pesos antes y después de cocinados. A diferencia del valor teórico estipulado, el porcentaje promedio obtenido en este estudio fue de 13.01\% (Cuadro 1), considerablemente bajo. Esto puede ser indicativo de que en las sodas en general se realiza un buen manejo de los alimentos a la hora de trocear, freír y cocinar. 
Finalmente, en el área de residuos de platos fue donde se presentó mayor cantidad de desperdicios en comparación con las otras tres áreas, existiendo diferencias significativas entre los datos ( $F=5.083, g . l=3, p$-value=0.0294). La soda 3 presentó el mayor porcentaje de PDA de $86.37 \%$, la soda 2 obtuvo el menor $54.70 \%$. Todas las sodas presentaron un porcentaje de PDA mucho mayor al estipulado en la literatura (figura 8). Además, el promedio general de todas las sodas es $69.37 \pm 13.15$, más alto que el brindado por la literatura (cuadro 1). Estos resultados indican que mucho del desperdicio de alimentos que se está viendo reflejado en el PDA general observado en la figura 7 es producto de esta área evaluada. Son los usuarios de las sodas los que están generando la mayor cantidad de desperdicios. El cual [12] puede responder a diversos factores, desde el tamaño de las porciones, la calidad de la comida, la composición dietaría de los alimentos entre otros.

A pesar de que la soda 3 tiene un alto porcentaje de PDA en esta área, se puede observar como en las otras dos áreas de la cocina casi no presenta pérdidas, esto gracias a las buenas prácticas que son implementadas por los funcionarios, sin embargo, los residuos de platos no pueden ser controlado por el personal de la soda, ya que es producto de la acción del usuario que consume el alimento. Por lo tanto, ésta es el área en el que se debe trabajar más, con el fin de concienciar a la población sobre el desperdicio de alimentos, por lo que se tiene proyectado trabajar en la sensibilización de esta población por medio de la campaña "Coma y disfruta sin dejar huella en el 2019" [7]. En [13] se recalca la importancia de generar campañas de concientización donde se explique a las personas que desperdiciar alimentos tiene un impacto no solo social sino también ambiental y económico, por lo que se debe ser tratado con celeridad. Para [14] existe una relación directa entre las campañas de sensibilización con la disminución en el desperdicio de alimentos, que es fundamental trabajar con los consumidores que generan residuos y desperdicios de alimentos.

Por otro lado, en cuanto a la producción diaria se obtuvo que existen diferencias en la cantidad de alimentos producidos por día en las cuatro sodas evaluadas de manera significativa ( $F=4.094$, g.l=3, p-value=0.0492), al igual que el porcentaje de PDA con respecto a esa producción diaria $(F=24.64, g . l=3, p-v a l u e=0.000215)$ (figura 9). En relación con esto, cada soda tiene diferente tipo de población de usuarios, por lo que algunas sodas producen más cantidad de alimentos que otras de acuerdo con la demanda de la cantidad de usuarios que los visitan [15].

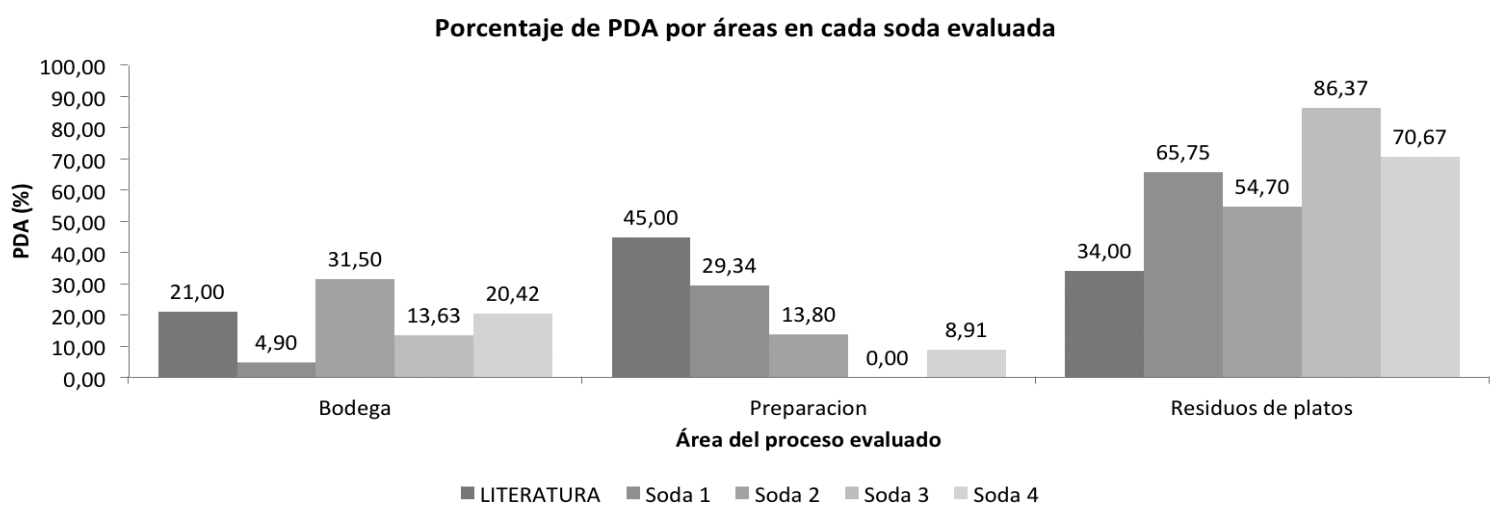

Figura 8. Porcentaje de PDA por áreas. 
Cuadro 1. PDA promedio en cada área evaluada.

\begin{tabular}{|c|c|c|}
\hline Área & Literatura (\%) & Promedio (\%) \\
\hline Bodega & 21 & $17.61 \pm 11.23$ \\
\hline Preparación & 45 & $13.01 \pm 12.29$ \\
\hline Residuos platos & 34 & $69.37 \pm 13.15$ \\
\hline
\end{tabular}

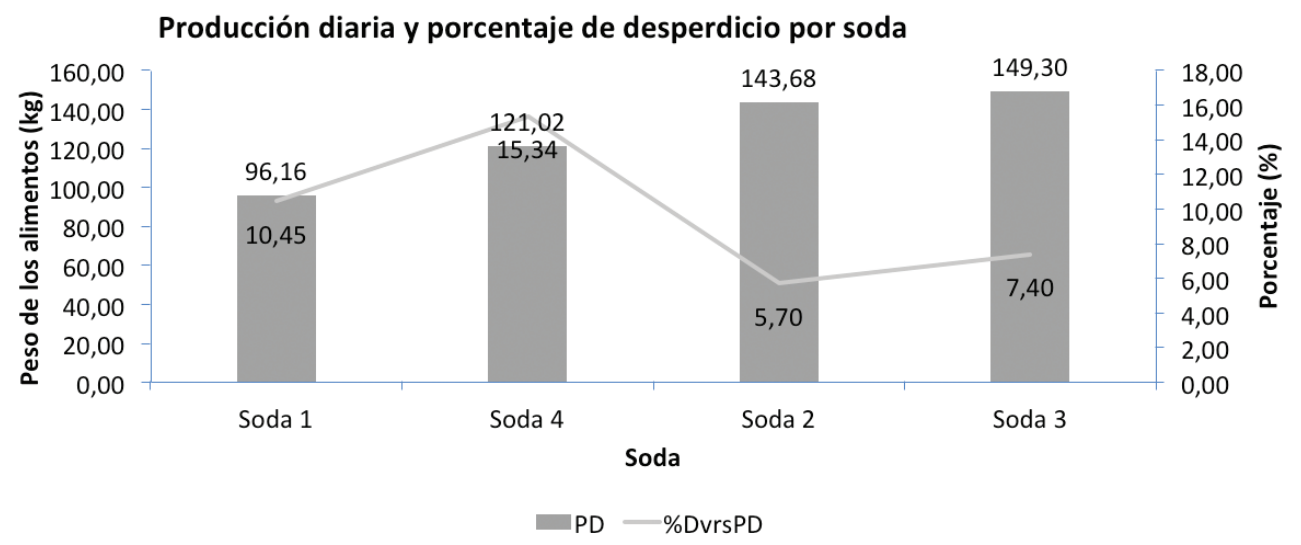

Figura 9. Producción diaria y porcentaje de desperdicio respecto a esa producción por soda.

Se puede observar como la soda 3 a pesar de ser la que produce mayor cantidad de comida $(149.30 \mathrm{~kg}$ ) presenta menos porcentaje de desperdicio de alimentos con respecto a su producción diaria $(7.40 \%)$. Cómo ya se mencionó, en esta soda existen buenas prácticas en el área de bodega y preparación por lo que el desperdicio que se percibe sea por situaciones que la soda no puede controlar como los desperdicios de los usuarios por devolución en platos.

La soda 4 por su parte tiene un alto porcentaje de desperdicio en relación con su producción diaria de alimentos (15.34\%). Esto pudo ser debido a una baja calidad de la comida por contener mucho aceite (ya que esta soda no entrega aceite residual a UNA Campus Sostenible). No se puede asegurar que en la soda se dé un sobreuso del aceite y que esté interfiriendo en la calidad de los alimentos, pero sí es un indicativo de que hay un mal manejo del aceite y éste puede estar captándose en la comida [18]. Esto puede ocasionar que se disminuya la calidad del alimento y que los usuarios dejen mucho desperdicio en los platos.

\section{Conclusiones y recomendaciones}

La medición de PDA en las distintas sodas del campus constituye un importante avance en el tema de desperdicios de alimentos que se relaciona a su vez con la cultura ambiental sostenible que la institución está comprometida en ser un modelo de gestión ambiental a nivel nacional por medio de la unidad de gestión ambiental UNA Campus Sostenible. Los resultados obtenidos demuestran el vacío que existe en este tema por parte de los estudiantes y usuarios que utilizan los servicios de alimentación, donde la mayor generación de PDA está dada por los desperdicios de los platos que estos usuarios dejan en los comedores.

A través de este estudio, se permite evidenciar una fuerte problemática en el desperdicio de residuos de platos en la universidad. En general con los desperdicios de residuos de platos se podría alimentar a 42 personas al año, lo que genera una alarma sobre qué se puede hacer para corregir esta práctica. Además, estos alimentos conllevan una importante inversión económica 
a la que no se le está poniendo la debida atención y que debe ser importante para autoridades de la institución. Es por esto como recomendación se recalca la necesidad de trabajar más en esta parte de la población estudiantil, pues a pesar de que para las sodas existe una preocupación por no desperdiciar alimento, la población cliente no tienen dicha sensibilización y conocimiento de los datos dan alarmantes. La creación de una campaña permitirá llegar hasta la población universitaria con el mensaje de sensibilizar sobre no desperdiciar alimentos y llegar a cero desperdicios en la Universidad Nacional.

También, es necesario que, por parte de las diversas autoridades de la institución, se profundice y se actúe más a fondo en el tema de Pérdidas y Desperdicios de Alimentos. Aspecto en el cual se está avanzando, al evidenciar a través de esta medición la existencia e identificación de la problemática. Para esto, lo más importante es lograr un óptimo trabajo en equipo, por parte de las diversas instancias y poder transmitir y sensibilizar a la población, e incentivar a realizar el cambio al preocuparse por el tema de Desperdicio de Alimentos, que al final no solo implica un coste económico sino también un costo ambiental.

Por otro lado, se debe capacitar al personal de las sodas, con la idea de generar un interés y sensibilización en este tema. Esto por medio de mejores prácticas y gestión de los alimentos como: verificar y mantener en constante seguimiento los productos prontos a vencer, aprovechar y emplear los diversos productos no tan agradables para la población meta, en otras recetas como una estrategia para no disponerlo y generar más residuo.

La metodología utilizada en este estudio es eficaz para determinar el desperdicio de alimentos, así como para conocer a lo interno las prácticas que tiene cada una de las diversas sodas al momento de preparar, ya que puede ser indicativo de más o menos desperdicio en las diferentes áreas de la cocina. Algunas de las sodas, crean e implementan buenas prácticas dentro de las áreas de la cocina en las que tienen control (bodega y preparación), sin embargo, se debe ser reforzado las sodas que presentaron deficiencia en estas áreas. Debido a las características propias de cada lugar es relevante analizar constantemente los sistemas de manejo y administración de las sodas en sus procesos de manipulación de alimentos y tasa de generación de desperdicios de alimentos.

\section{Referencias}

[1] Organización de las Naciones Unidas para la Alimentación y la Agricultura. FAO (2016) Pérdidas y Desperdicios de Alimentos en América Latina y el Caribe. Disponible en: www.fao.org/publications

[2] Red Costarricense para la disminución y Pérdida de Desperdicios de Alimentos (PDA). (2017). Disminución de Pérdidas y desperdicios de alimentos. Guía para mediciones, cocinas institucionales y comerciales. Tecnológico de Costa Rica, Costa Rica. 33p.

[3] Organización de las Naciones Unidas. (s.f) Objetivos de Desarrollo Sostenible- 17 Objetivos para transformar nuestro mundo. Página Web Principal. Disponible en https://www.un.org/sustainabledevelopment/es/sustainable-development-goals/

[4] Organización de las Naciones Unidas para la Alimentación y la Agricultura. (2017) Comité de Seguridad Alimentaria Mundial: Marcar la diferencia en la Seguridad Alimentaria y la Nutrición. Disponible en: http://www. fao.org/cfs/es/

[5] Organización de las Naciones Unidas para la Alimentación y la Agricultura. (2015) Comité de Seguridad Alimentaria Mundial: Marco de Acción para la Seguridad Alimentaria y la Nutrición en Crisis Prolongadas. Disponible en http://www.fao.org/cfs/cfs-home/products/es/

[6] Ley para la Gestión Integral de Residuos, N8839 del 13 de Julio de 2010. (2012). Reglamento General a la Ley para la Gestión Integral de Residuos. Programa CYMA. Competitividad y Medio Ambiente. San José, Costa Rica. Disponible en

[7] J. Rojas, comunicación personal, octubre 2018.

[8] WRAP. Hospitality and Food Service Reducing Food Waste: Starting Off. UK. 2014. 
[9] M. Rodríguez, "Estimación de las pérdidas de alimentos en una industria del sector lácteo y sus posibles efectos sobre la seguridad alimentaria y nutricional" M.S. tesis, Departamento de nutrición humana, Universidad Nacional de Colombia, Bogotá, 2016. Disponible: http://bdigital.unal.edu.co/55497/13/marioalejandrorodriguezlopez.2016.pdf

[10] D. Suarez, "Análisis socioeconómico del desperdicio de alimentos para el consumo humano generado por el Supermercado la Canasta de la ciudad de Cucuta". Trabajo de grado, Facultad de Ciencias económicas administrativas y contables, Universidad Libre, Cucuta Norte Santander, 2017. Disponible: https:// repository.unilibre.edu.co/bitstream/handle/10901/11461/ANALISIS\%20SOCIOECNOMICO\%20DEL\%20 DESPERDICIO\%20DE\%20ALIMENTOS\%2OPARA\%20EL\%2OCONSUMO\%2OHUMANO\%20GENERADO\%20 POR\%20EL\%20SUPERMECADO\%20LA\%20CANASTA\%20DE\%20LA\%20CIUDAD\%20DE\%20CUCUTA. pdf?sequence $=1$ \&isAllowed $=\mathrm{y}$

[11] E. Basso et al., "Valoremos los alimentos, evitemos pérdidas y desperdicios", Revista DIAETA, vol. 34, no. 155, pp. 25-32. 2016. Disponible en: http://www.scielo.org.ar/pdf/diaeta/v34n155/v34n155a04.pdf

[12] F. Cedeño, "El desperdicio de alimentos: una perspectiva desde los estudiantes de Administración de Empresas de la UPS Guayaquil", Revista Retos, no. 11, pp. 53-66, 2016 Disponible en: https://www.dspace. ups.edu.ec/bitstream/123456789/13916/1/ret_n11_Franco.pdf

[13] Y. Chiriboga, (2018). Campaña de publicidad social para evitar el desperdicio de comida a través de donación de alimentos: Caso Banco de alimentos de Quito. Disponible en: http://dspace.udla.edu.ec/bitstream/33000/8764/1/UDLA-EC-TPU-2018-05.pdf

[14] M. Bustamante, A. Alfonso, I. De los ríos, (2018). Desperdicio alimentario en comedores escolares, cuantificación e identificación de posibles factores condicionantes. Disponible en: https://www.fesbal.org/wp-content/ uploads/2018/10/Informe_FESBAL_Comedores_Sept_2018.pdf

[15] C.A. Fernández, "La gestión integral de los residuos sólidos urbanos en el desarrollo sostenible local", Revista Cubana de Química, vol. 17, no. 3, 35-39, 2005. Recuperado de http://www.redalyc.org/ $\mathrm{html} / 4435 / 443543687013 /$

[16] B. Lipinski, et al. (2013). Reducing Food Loss and Waste. World Resources Institute. pp. 1-40.

[17] V.L. González, "El insostenible desperdicio de alimentos: ¿Qué podemos hacer los consumidores?”, Revista CESCO de Derecho de Consumo, no. 14, pp. 203-216, 2015. Disponible en: https://cesco.revista.uclm.es/ index.php/cesco/article/view/801

[18] K. López-Cruz, J. Rojas-Vargas, J. Bogantes-Sánchez, "Gestión integral de los residuos de aceite vegetal de cocina en las sodas del Campus Omar Dengo de la Universidad Nacional de Costa Rica", Uniciencia, vol. 22, no. 1, pp. 18-29, 2018. 